\title{
Mapping the Damping Dynamics of Mega-Ampere Electron Pulses Inside a Solid
}

\author{
Moniruzzaman Shaikh, ${ }^{1}$ Amit D. Lad, ${ }^{1}$ Gabriele Birindelli, ${ }^{2}$ Kevin Pepitone, ${ }^{3}$ Jagannath Jha, ${ }^{1}$ Deep Sarkar, \\ Sheroy Tata, ${ }^{1}$ Gourab Chatterjee, ${ }^{1}$ Indranuj Dey, ${ }^{1}$ Kamalesh Jana, ${ }^{1}$ Prashant K. Singh, ${ }^{1}$ \\ Vladimir T. Tikhonchuk, ${ }^{2}$ P. P. Rajeev, ${ }^{4, *}$ and G. Ravindra Kumar ${ }^{1, \dagger}$ \\ ${ }^{1}$ Tata Institute of Fundamental Research, 1 Homi Bhabha Road, Mumbai 400005, India \\ ${ }^{2}$ Centre Lasers Intenses et Applications, University of Bordeaux-CNRS-CEA, Talence 33405, France \\ ${ }^{3}$ CERN, Beam Department (BE), Geneva 1211, Switzerland \\ ${ }^{4}$ Central Laser Facility, Rutherford Appleton Laboratory, Oxfordshire QX11 OQX, United Kingdom
}

(Received 21 August 2017; revised manuscript received 20 November 2017; published 5 February 2018)

\begin{abstract}
We report the lifetime of intense-laser $\left(2 \times 10^{19} \mathrm{~W} / \mathrm{cm}^{2}\right)$ generated relativistic electron pulses in solids by measuring the time evolution of their Cherenkov emission. Using a picosecond resolution optical Kerr gating technique, we demonstrate that the electrons remain relativistic as long as 50 picoseconds-more than 1000 times longer than the incident light pulse. Numerical simulations of the propagation of relativistic electrons and the emitted Cherenkov radiation with Monte Carlo GEANT4 package reproduce the striking experimental findings.
\end{abstract}

DOI: 10.1103/PhysRevLett.120.065001

Multiterawatt femtosecond lasers can accelerate electrons on a solid surface to near-light speeds. Just like the driving laser field, these electrons appear in ultrashort pulses, launching mega-ampere peak currents into the solid material. These are among the largest current pulses ever created in the laboratory and their transport in the target involves complex physics [1-7]. A major problem in understanding the physics arises due to the paucity of information on the characteristics of these fast electrons as they transit through the solid. From a practical viewpoint, the behavior of energetic electrons as they pass through a dense, hot solid is central to a multitude of applications of short-pulse laser-matter interaction [8,9], as the energy is transferred from the driving laser field to the secondary emissions through these electrons. Although hot-electron transport through solids has been studied [10-13], very little is known about the time the electrons spend inside a solid while dissipating their energy. In particular, their "transit time" effectively determines the efficiency of energy transfer and the duration of emissions arising from the interaction. There have, however, been some attempts to measure dynamics of energetic electrons at rear of thin targets using (a) time-resolved reflectivity [14], (b) interferometry [15], or (c) measurement of the electric field [16]. There have also been studies of the dynamics inside the target using X-ray spectroscopy [17]. However, there are no

Published by the American Physical Society under the terms of the Creative Commons Attribution 4.0 International license. Further distribution of this work must maintain attribution to the author(s) and the published article's title, journal citation, and DOI. reliable methods till date for unambiguously mapping the transit time of relativistic electrons through dense media. In this Letter, we report "real-time" measurements of the transition of relativistic electrons through a solid dielectric by monitoring their Cherenkov emission.

We employ an ultrafast gating technique based on the optical Kerr effect [18] to map the evolution of the Cherenkov emission with a picosecond temporal resolution. From a direct measurement of the duration of the Cherenkov emission, we deduce the lifetime of energetic electrons inside the target. We find that the lifetime of electrons, even at relativistic energies, is in good agreement with collisional damping models in a solid. A qualitative model is presented that helps in understanding the dissipation process of energetic electrons inside dense media.

The experiment (Fig. 1) was performed with a 100terawatt Ti:sapphire laser. $P$-polarized laser pulses of $25 \mathrm{fs}$ duration (central wavelength at $800 \mathrm{~nm}$ and $1.0 \mathrm{~J}$ pulse energy) were focused with a $f / 3$ off-axis parabolic mirror to a $15-\mu \mathrm{m}$ spot at near-normal incidence $\left(2^{\circ}\right)$, creating a peak intensity of $2 \times 10^{19} \mathrm{~W} / \mathrm{cm}^{2}$. We used transparent dielectric targets (BK7 glass, refractive index $n=1.5$ at $\lambda=560 \mathrm{~nm}$ ) with $\mathrm{Al}$ coatings of $200-\mathrm{nm}$ thickness at the front of the targets. Optical Kerr rotation in carbon disulphide $\left(\mathrm{CS}_{2}\right)$ was employed to gate the Cherenkov emission from the targets in a time window of $2 \mathrm{ps}$, as shown in Fig. 1(d). We observed Cherenkov spectra in the 300-700 nm range for all targets (see Supplemental Material [19]).

Cherenkov emission is produced in a cone with an angle $\theta\left(\cos \theta=c / v_{e} n\right)$ by electrons propagating with a superluminal speed $v_{e}>c / n$ in a medium, where $c$ is the light speed in vacuum [20-24]. In BK7 glass, electrons with 


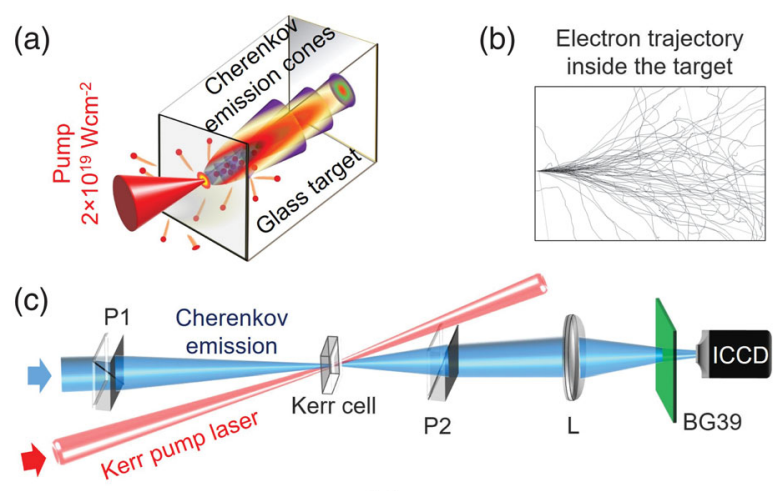

(d)

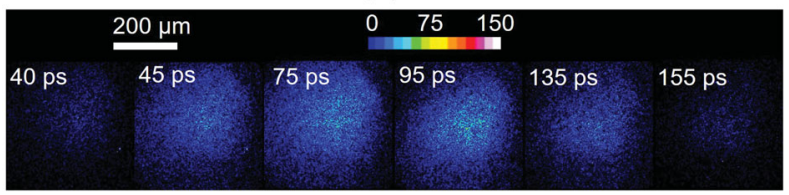

FIG. 1. (a) Schematic of laser solid interaction and generation of Cherenkov emission. (b) Simulation of electron trajectories inside the target. (c) Schematic of optical Kerr gate: $L$-achromatic lens $(f=20 \mathrm{~cm}) ; P 1, P 2$ : Glan-air polarizers; Kerr cell: $\mathrm{CS}_{2}$ as the nonlinear Kerr medium driven by a pick-off laser beam; BG 39: bandpass filter; ICCD: intensified charged coupled device camera. (d) Snapshots of Cherenkov emission from a 10-mm BK7 glass target.

speeds $v_{e}>2 \times 10^{8} \mathrm{~m} / \mathrm{s}$ (electron energies larger than $E_{\min }=174 \mathrm{keV}$ ) emit Cherenkov radiation. The higher the electron energy, the larger the cone angle. However, the solid angle of our imaging system limits the detectable directions in our experiment. The half angle of acceptance was $14^{\circ}$, accounting for glass refractive index, enabling collection of emission at angles less than $9^{\circ}$. These angles define a detectable energy range of $189 \pm 10 \mathrm{keV}$ for hot electrons propagating along the normal inside the BK7 target. However, this range broadens when the electrons deviate from the paraxial propagation due to scattering.

The laser-solid interaction produces hot electrons spread over a broad energy range [1], but their energy distribution inside a solid is difficult to assess. So far, most studies have used micron-thick targets and relied on optical transient emission from the rear side to diagnose hot-electron transport $[11,23,24]$. In relativistic laser-matter interaction, however, electron energies can be as high as a few $\mathrm{MeV}$, with a stopping range of a few millimetres in a solid [9]. For thin targets, only a fraction of the hot-electron population with the stopping range matching the target thickness would contribute to the Cherenkov emission, while the higher energy electrons would escape the target. Moreover, it is well known that these electrons do not travel in a straight line through solids, as they suffer multiple elastic collisions with ions and lose energy in collisions with atomic electrons [Fig. 1(b)]. Electrons with initial speeds barely above the light speed in the medium would cease to emit Cherenkov radiation after the first scattering event. In contrast, higher-energy electrons may penetrate deeper into the target, gradually losing energy and continuing Cherenkov emission until their energy falls below the threshold value $E_{\min }$. Therefore, the initial population of high-energy electrons would eventually contribute to the observed Cherenkov emission as soon as the energies are damped down to the experimentally detectable window.

Measuring the Cherenkov emission as a function of target thickness [Fig. 2(a)] can yield information on the energy distribution of the hot-electron population. We see that the emission increases initially and saturates beyond $10 \mathrm{~mm}$ thickness. This clearly shows the contribution of electrons with energies much higher than $189 \mathrm{keV}$ in the observed emission, because electrons up to this energy will be stopped in the first few hundred microns of the target. Further, since the emission saturates, it is clear that all energetic electrons produced at the front surface are retarded sufficiently at a distance of $10 \mathrm{~mm}$, so that there are no electrons with energies higher than $174 \mathrm{keV}$ beyond this length. The stopping range of electrons $[25,26]$ is known to be inversely proportional to the mass density $\rho$. A 10 -mm-thick BK7 target $(\rho=2.5 \mathrm{~g} / \mathrm{cc})$ stops electrons with initial energy less than $5 \mathrm{MeV}$. This presents the cutoff energy for electrons for our interaction conditions, in good agreement with other experimental observations [1]. These electrons produce Cherenkov emission throughout the length of the target: electrons with low energies of a few hundred $\mathrm{keV}$ have a stopping range of less than $1 \mathrm{~mm}$ and, thus, emit Cherenkov radiation near the target front for a short time, while the electrons with higher energies propagate deeper with a superluminal speed and radiate the Cherenkov photons in the detectable range near the target rear. Dependence of Cherenkov emission on target thickness thus becomes a good diagnostic tool for monitoring the generation and transport of the hot-electron population produced in laser-solid interaction.

In order to measure the cooling time of the hot-electron population, we examine the temporal characteristics of Cherenkov emission. We employ an optical Kerr gate, driven by a laser pulse synchronized to arrive within the (a)

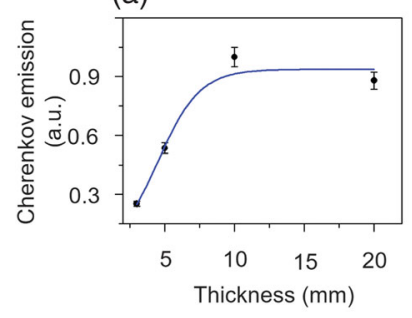

(b)

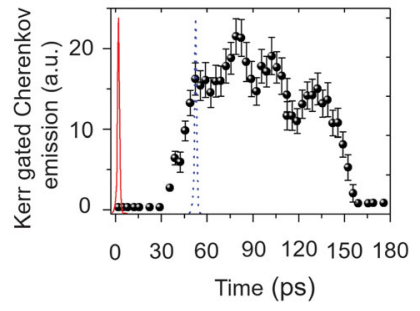

FIG. 2. Cherenkov emission from a 10-mm BK7 glass target. (a) Spatially and temporally integrated emission as a function of target thickness. (b) Spatially integrated, Kerr-gated, signal as a function of delay. The red curve indicates time zero, $t=0$. The blue dashed line shows the main laser pulse reaching the gate. 
5-ns time window of the intensified charge coupled device camera, in order to temporally slice the emission. Figure 2(b) shows the temporal evolution of the spatially integrated Cherenkov emission from a $10-\mathrm{mm}$ target with respect to the instant of arrival of the laser pulse at the target front, called "time zero" $(t=0)$. The emission rises to the first peak in about $20 \mathrm{ps}$ (from 33 to $53 \mathrm{ps}$ ), followed by a broad saturation for another $20 \mathrm{ps}$, and decays gradually over the next $60-80 \mathrm{ps}$. Thus, the total time of the detected Cherenkov emission is more than 120 ps (from 33 to 153 ps), over 5000 times longer than the hot-electron pulse entering the target. This large difference is caused by (i) the time needed for an electron to reduce its initial energy to the threshold of the Cherenkov emission $E_{\min }$, (ii) a ballistic spread of electrons in the target generating extra path lengths, and (iii) the time delay of the photons emitted at different distances from the source.

Since the Cherenkov-emitting electrons travel faster than light in target, the emission from the low-energy electrons reaches the optical Kerr gate later than the Cherenkov photons from the higher-energy electrons. Thus, emissions from highest-energy electrons appear at earlier times, followed by emissions from electrons with lower initial energies. Therefore, the observed temporal evolution of the Cherenkov emission provides an inverse energy map of the hot-electron distribution inside the solid.

The observed Cherenkov temporal evolution has three distinct features: a "rise," a "broad peak," and a "decay." The earliest photons we measure come from a few highestenergy electrons that propagate without losing much of their energy until the target rear, where they get scattered off nuclei at angles of $\sim 40^{\circ}-50^{\circ}$ [9]. As these electrons travel at near-light speeds until target rear, the Cherenkov photons they generate are produced at time $t_{1} \simeq d / c$ with respect to $t=0, d$ being the target thickness. Similarly, a small population of electrons with lower energies that do not get damped, yet undergo large angle elastic scattering throughout the length of the target at various depths, emit Cherenkov photons at times later than $t_{1}$.

Hot electrons produced in femtosecond laser-solid interactions are typically modeled with a Maxwellian distribution with a temperature depending on the laser intensity [1]. Such an exponential decrease of a number of electrons with energy explains the initial rise of the Cherenkov signal. In a Maxwellian distribution, it is reasonable to expect that the maximum of Cherenkov emission would come from electrons with energy $E \gtrsim E_{\min }=174 \mathrm{keV}$. The stopping range of the electrons just above this energy is very small, $\sim 200 \mu \mathrm{m}[25,26]$, so the Cherenkov photons generated by them need to propagate through the entire target before arriving at the target rear. Since photons travel at a slower velocity, $v_{p}=c / n$, through the target with refractive index $n$, these photons reach the target rear at time $t_{2}=n d / c$. The difference between $t_{1}$ and $t_{2}$ defines the rise time, $t_{r}=(n-1) d / c$.
The majority of hot electrons, however, lose energy as they propagate through the target. The electron cooling time in target can be estimated with the electron stopping power $d E_{e} / d s \simeq 0.5 \mathrm{MeV} / \mathrm{mm}[25,26]$ and the average electron velocity $\bar{v}_{e} \simeq 0.8 c$ : the product $\bar{v}_{e} d E_{e} / d s=$ $d E_{e} / d t \simeq 0.12 \mathrm{MeV} / \mathrm{ps}$ defines the electron cooling rate. For maximum energy $E_{\max }$, we estimate electron lifetime as $t_{e}=E_{\max } /\left(d E_{e} / d t\right)$. This means that Cherenkov photons are emitted inside the target during the time $t_{e}$, but the time of their arrival to the target rear depends on their emission point. The first photons which need not travel through the target arrive at time $t_{1}$. The last one generated at the front side arrives at $t_{e}+t_{2}$. Depending on the laser intensity, the target material and thickness, $t_{e}$, can be greater or smaller than $t_{2}-t_{1}$; for a BK7 target of $d=10 \mathrm{~mm}$ thickness, we have $t_{1} \simeq 33 \mathrm{ps}$, that is, we start seeing the first Cherenkov photons at around $33 \mathrm{ps}$ from $t=0$. This is consistent with the experimental observation [Fig. 2(b)]. With $n=1.5$ and $E_{\max }=5 \mathrm{MeV}$, we get $t_{2}=50 \mathrm{ps}$ and $t_{e}=42 \mathrm{ps}$. This means the last electron reaching the rear surface would have cooled down below the Cherenkov threshold before the photons from the front surface arrive. The rise time of the Cherenkov signal therefore would be $t_{2}-t_{1} \simeq 17 \mathrm{ps}$, which again agrees with the data in Fig. 2(b).

This estimates the maximum expected signal duration to be $t_{2}-t_{1}+t_{e}=59 \mathrm{ps}$, because no superluminal electrons are left in the target beyond this time. In order to corroborate the lifetime of superluminal electrons, we undertake Monte Carlo simulations of the electron propagation in glass, using the MCNPX [27] code (see Supplemental Material [19]). The energy and angular distribution of hot electrons relevant to our experimental conditions have been studied extensively $[1,11,28,29]$. For the laser pulse intensity of $\sim 10^{19} \mathrm{~W} / \mathrm{cm}^{2}$, about $30 \%$ of the incident laser energy is converted into hot electrons with energies of a few hundred $\mathrm{keV}$, ejected in a cone with the opening angle of $\sim 30^{\circ}$, with a relatively small (less than $1 \%$ ) number injected in the direction normal to the target surface as a narrow jet having an effective temperature of $\sim 1 \mathrm{MeV}$. In this calculation, we look at the evolution of a single bunch of $5-\mathrm{MeV}$ electrons propagating through the medium experiencing collisions with atomic nuclei and bound electrons.

We examine the depth of electron penetration and the time of electron slowing down. Figures 3(a) and 3(b) show the electron distribution and its temporal evolution at two representative distances inside the target. At a given target depth, the electrons appear with time delay related to their propagation time, about $15 \mathrm{ps}$ for $4.4 \mathrm{~mm}$ and $25 \mathrm{ps}$ for $6.6 \mathrm{~mm}$. The width of electron distribution increases and their number decreases with time. It is important to note that the position of the peak of electron distribution decreases linearly with time, $d E_{\max } / d t \simeq 0.11 \mathrm{MeV} / \mathrm{ps}$ being the rate of energy loss. Remarkably, this rate is independent of the initial electron energy. The electrons at a 

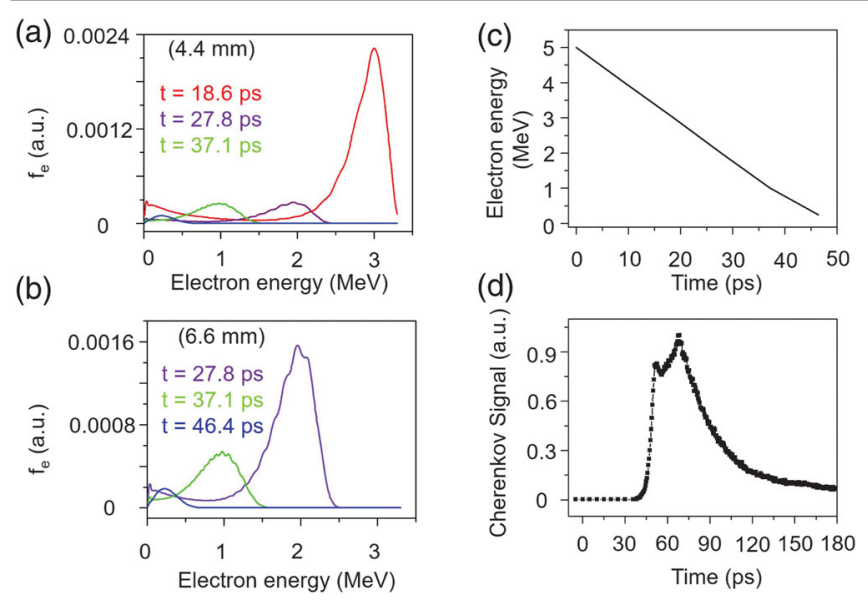

FIG. 3. Numerical simulations of relativistic electron transport inside the BK7 target. (a),(b) Temporal evolution of the electron distribution function at a depth of (a) $4.4 \mathrm{~mm}$ and (b) $6.6 \mathrm{~mm}$ at time instants of $18.6 \mathrm{ps}$ (red), $27.8 \mathrm{ps}$ (violet), $37.1 \mathrm{ps}$ (green), and $46.4 \mathrm{ps}$ (blue) for the case of a $5-\mathrm{MeV}$ monoenergetic electron bunch. (c) Temporal evolution of the maximum (5 MeV) electron energy. (d) GEANT4 simulation of Cherenkov emission from a 10mm-thick BK7 target.

certain depth follow the curve shown in Fig. 3(c), starting from the maximum energy at the moment of injection. It is clear that no superluminal electrons are left in the target after $46 \mathrm{ps}$, which is in agreement with the qualitative estimate presented earlier and with the maximum emission time in the BK7 glass target shown in Fig. 2.

We see that the Cherenkov signal lasts over $100 \mathrm{ps}$ even though the superluminal electrons do not survive that long. In order to verify this, we performed integrated Monte Carlo simulations of the electron propagation and Cherenkov emission from a 10-mm BK7 target with GEANT4 [30]. We present here a simple but representative case of a monoenergetic, collimated electron beam at $5 \mathrm{MeV}$ entering the target. The temporal profile of the Cherenkov signal is not sensitive to the initial divergence and the energy distribution of electrons as the most energetic electrons define the time of photon emission (see Supplemental Material [19]). The Cherenkov photons between 380 and $730 \mathrm{~nm}$ collected at the end of the target were counted. The temporal evolution of the Cherenkov signal is shown in Fig. 3(d). The simulated emission is observed to last over 120 ps, just as observed in experiments.

As seen from these simulations, the discrepancy between the lifetimes of superluminal electrons and the Cherenkov emission is due to the multiple scattering events the electrons undergo while getting damped [9,31], spreading them in the transverse direction. Cherenkov photons from these sources will need to traverse additional paths in order to reach the detector, yielding delays in the observed Cherenkov signal. Further, the multiple scattering of electrons by nuclei-Mott electron scattering [31]—produces an angular spread yielding electrons propagating even in the opposite direction with energies above $E_{\min }$ [Fig. 1(b)]. These electrons will emit Cherenkov photons towards the front surface, which return to the rear side after reflection from the aluminium layer in front, incurring a maximum delay $2 t_{2}=2 \mathrm{nd} / \mathrm{c}$, which is $100 \mathrm{ps}$ in the present case. The total duration of the Cherenkov pulse thus becomes $t_{d}=t_{e}+2 t_{2}-t_{1}=$ $E_{\max } /\left(d E_{e} / d t\right)+(2 n-1) d / c$.

The simple estimates given above can explain the experimental observations. For a 10-mm-thick BK7 target, we obtain $t_{d} \simeq 110 \mathrm{ps}$, in excellent agreement with the experimental data in Figs. 2(a) and 2(b). The generality of these explanations is confirmed by extending them to the experimental observations of temporal evolution of Cherenkov emission from a thinner target ( $5 \mathrm{~mm}, \mathrm{BK} 7$ ) and a glass of higher density and refractive index (LASF35) (Figs. 2 and 3 of the Supplemental Material [19]). The risetime estimates from the model agree with experimental observations in both cases. This qualitative reasoning gives us direct access to the electron cooling time $t_{e}$ and the cooling rate, provided the maximum electron energy is known.

While our numerical simulations explain the main features of the temporal evolution which are related to the longitudinal distribution of the photon emission inside the target, it is likely that the multiple peaks observed arise from their transverse spread as well as multiple photon bouncing within the target. The transverse spread resulting from multiple collisions as well as reflections from the target front and the ionization front that keeps moving towards the target rear can result in nonuniform, extended sources within the target. Preliminary investigations of the effect of such extended sources using the ray-tracing simulation program COMSOL indicate that multiple peaks can appear in the temporal evolution of Cherenkov emission (see Supplemental Material [19]). Further studies, which are currently underway, are required to understand this phenomenon fully.

In conclusion, we directly map the temporal evolution of superluminal electrons inside a solid as they transfer energy to the lattice, using an ultrafast gating of their Cherenkov emission. Superluminal electrons continue to dissipate energy for tens of picoseconds-over 1000 times longer than electron pulses that enter the solid. A simple model based on collisional damping can explain the results satisfactorily because the current of fast electrons is relatively low. Along with unravelling the temporal dynamics of energy transfer, the work reported here also gives a handle on the depth-dependent energy distribution of electrons. Further development of this method will allow us to directly measure the energy distribution of electrons inside a solid at any given position. Direct measurements of electron interaction time scales provides a major missing link in the understanding of hot-electron transport through solids that forms the crux of high-energy-density science. 
These measurements may trigger new thinking on fastelectron transport in dense media and are vital for the applications that rely on the duration and efficiency of energy transfer from electrons to solids.

We acknowledge valuable discussions with Robert Bingham. G. R. K. acknowledges partial support from J. C. Bose Fellowship Grant No. JCB-37/2010 of the Department of Science and Technology, Ministry of Science and Technology, Government of India. G. R. K. and P. P. R thank the Newton-Bhabha UK-India programme for facilitating this collaboration.

*rajeev.pattathil@stfc.ac.uk †rk@tifr.res.in

[1] P. Gibbon, Short Pulse Laser Interactions with Matter-An Introduction (Imperial College Press, London, 2005).

[2] V. T. Tikhonchuk, Phys. Plasmas 9, 1416 (2002).

[3] J. R. Davies, Phys. Rev. E 68, 037501 (2003).

[4] S. I. Krasheninnikov, A. V. Kim, B. K. Frolov, and R. Stephens, Phys. Plasmas 12, 073105 (2005).

[5] A. Debayle and V. T. Tikhonchuk, Phys. Rev. E 78, 066404 (2008).

[6] O. Klimo, V. T. Tikhonchuk, and A. Debayle, Phys. Rev. E 75, 016403 (2007).

[7] H. Alfven, Phys. Rev. 55, 425 (1939).

[8] M. Tabak, J. Hammer, M. E. Glinsky, W. L. Kruer, S. C. Wilks, J. Woodworth, E. M. Campbell, and M. D. Perry, Phys. Plasmas 1, 1626 (1994).

[9] J. Caron, J. L. Feugeas, B. Dubroca, G. Kantor, C. Dejean, G. Birindelli, T. Pichard, P. Nicola, E. d'Humires, M. Frank, and V. Tikhonchuk, Phys. Med. 31, 912 (2015).

[10] H. Liu, C. M. Carpenter, H. Jiang, G. Pratx, C. Sun, M. P. Buchin, S. S. Gambhir, L. Xing, and Z. Cheng, J. Nucl. Med. 53, 1579 (2012).

[11] J. J. Santos, A. Debayle, Ph. Nicola, V. Tikhonchuk, M. Manclossi, D. Batani, A. Guemnie-Tafo, J. Faure, V. Malka, and J. J. Honrubia, Phys. Plasmas 14, 103107 (2007).

[12] P. McKenna, A. P. L. Robinson, D. Neely, M. P. Desjarlais, D. C. Carroll, M. N. Quinn, X. H. Yuan, C. M. Brenner, M. Burza, M. Coury, P. Gallegos, R. J. Gray, K. L. Lancaster, Y. T. Li, X. X. Lin, O. Tresca, and C. G. Wahlström, Phys. Rev. Lett. 106, 185004 (2011).

[13] M. N. Quinn, X. H. Yuan, X.X. Lin, D. C. Carroll, O. Tresca, R. J. Gray, M. Coury, C. Li, Y. T. Li, C. M. Brenner, A. P. L. Robinson, D. Neely, B. Zielbauer, B. Aurand, J. Fils, T. Kuehl, and P. McKenna, Plasma Phys. Controlled Fusion 53, 025007 (2011).
[14] J. S. Green, C. D. Murphy, N. Booth,a R. J. Dance, R. J. Gray, D. A. MacLellan, P. McKenna, D. Rusbya, and L. Wilson, J. Instrum. 9, P03003 (2014).

[15] P. Antici, S. N. Chen, L. Gremillet, T. Grismayer, P. Mora, P. Audebert, and J. Fuchs, Rev. Sci. Instrum. 81, 113302 (2010).

[16] R. Pompili, M. P. Anania, F. Bisesto, M. Botton, M. Castellano, E. Chiadroni, A. Cianchi, A. Curcio, M. Ferrario, M. Galletti, Z. Henis, M. Petrarca, E. Schleifer, and A. Zigler, Sci. Rep. 6, 35000 (2016).

[17] R. Shepherd, P. Audebert, R. Booth, B. Young, J. Bonlie, D. Nelson, S. Shiromizu, D. Price, D. Norman, J. Dunn, K. Widmann, and P. Springer, Rev. Sci. Instrum. 75, 3765 (2004).

[18] D. R. Symes, U. Wegner, H.-C. Ahlswede, M. J. V. Streeter, P. L. Gallegos, E. J. Divall, R. A. Smith, P. P. Rajeev, and D. Neely, Appl. Phys. Lett. 96, 011109 (2010).

[19] See Supplemental Material at http://link.aps.org/ supplemental/10.1103/PhysRevLett.120.065001 for (i) typical spectrum of Cherenkov emission, (ii) Temporal evolution of Cherenkov emission from $5 \mathrm{~mm} \mathrm{BK} 7$ and (iii) $9 \mathrm{~mm}$ LASF35 target, (iv) hot electron source for MCNPX simulation, (v) details for GEANT4 simulation and (vi) details of ray-tracing simulation.

[20] G. S. Smith, An Introduction to Classical Electromagnetic Radiation (Cambridge University Press, Cambridge, England, 1997), p. 411.

[21] F. Brandl, G. Pretzler, D. Habs, and E. Fill, Europhys. Lett. 61, 632 (2003).

[22] J. Zheng, C. X. Yu, Z. J. Zheng, and K. A. Tanaka, Phys. Plasmas 12, 093105 (2005).

[23] M. Manclossi, J. J. Santos, D. Batani, J. Faure, A. Debayle, V. T. Tikhonchuk, and V. Malka, Phys. Rev. Lett. 96, 125002 (2006).

[24] H. Habara, K. Ohta, K. A. Tanaka, G. R. Kumar, M. Krishnamurthy, S. Kahaly, S. Mondal, M. K. Bhuyan, R. Rajeev, and J. Zheng, Phys. Rev. Lett. 104, 055001 (2010).

[25] L. Katz and A. S. Penfold, Rev. Mod. Phys. 24, 28 (1952).

[26] C. D. Zerby and F. L. Keller, Nucl. Sci. Eng. 27, 190 (1967).

[27] MCNPX Users Manual, Version 2.7.0, LA-CP-11-004 38 (Los Alamos National Laboratory, 2011).

[28] G. Malka and J. L. Miquel, Phys. Rev. Lett. 77, 75 (1996).

[29] J. S. Green et al., Phys. Rev. Lett. 100, 015003 (2008).

[30] J.Allison et al., Nucl. Instrum. Methods Phys. Res., Sect. A 835, 186 (2016).

[31] N. F. Mott and H. S. W. Massey, The Theory of Atomic Collisions, 3rd ed. (Oxford University Press, Oxford, 1965). 